\title{
Pain assessment tools in paediatric palliative care: A systematic review of psychometric properties and recommendations for clinical practice
}

Palliative Medicine

2022, Vol. 36(1) 30-43

(C) The Author(s) 2021

(c) (i)

Article reuse guidelines:

sagepub.com/journals-permissions

DOI: $10.1177 / 02692163211049309$

journals.sagepub.com/home/pmj

(SAGE

\author{
Adrienne YL Chan ${ }^{1,2,3}$, Mengqin Ge ${ }^{1}$, Emily Harrop ${ }^{4,5}$, \\ Margaret Johnson $^{6}$, Kate Oulton ${ }^{7}$, Simon S Skene ${ }^{8}$ iD, \\ Ian CK Wong ${ }^{1,2,9,10}$, Liz Jamieson ${ }^{9,10}$ iD, Richard F Howard7 (iD \\ and Christina Liossi ${ }^{7,11}$ iD
}

\begin{abstract}
Background: Assessing pain in infants, children and young people with life-limiting conditions remains a challenge due to diverse patient conditions, types of pain and often a reduced ability or inability of patients to communicate verbally.

Aim: To systematically identify pain assessment tools that are currently used in paediatric palliative care and examine their psychometric properties and feasibility and make recommendations for clinical practice.

Design: A systematic literature review and evaluation of psychometric properties of pain assessment tools of original peer-reviewed research published from inception of data sources to April 2021.

Data sources: PsycINFO via ProQuest, Web of Science Core, Medline via Ovid, EMBASE, BIOSIS and CINAHL were searched from inception to April 2021. Hand searches of reference lists of included studies and relevant reviews were performed.

Results: From 1168 articles identified, 201 papers were selected for full-text assessment. Thirty-four articles met the eligibility criteria and we examined the psychometric properties of 22 pain assessment tools. Overall, the Faces Pain Scale-Revised (FPS-R) had high cross-cultural validity, construct validity (hypothesis testing) and responsiveness; while the Faces, Legs, Activity, Cry and Consolability (FLACC) scale and Paediatric Pain Profile (PPP) had high internal consistency, criterion validity, reliability and responsiveness. The number of studies per psychometric property of each pain assessment tool was limited and the methodological quality of included studies was low.

Conclusion: Balancing aspects of feasibility and psychometric properties, the FPS-R is recommended for self-assessment, and the FLACC scale/FLACC Revised and PPP are the recommended observational tools in their respective age groups.
\end{abstract}

\section{Keywords}

Pain measurement, pain assessment, palliative care, paediatrics

\footnotetext{
${ }^{1}$ Centre for Safe Medication Practice and Research, Department of Pharmacology and Pharmacy, Li Ka Shing Faculty of Medicine, The University of Hong Kong, Pok Fu Lam, Hong Kong

${ }^{2}$ Laboratory of Data Discovery for Health (D24H), Hong Kong Science Park, Pak Shek Kok, Hong Kong

${ }^{3}$ Groningen Research Institute of Pharmacy, Unit of Pharmacotherapy, Epidemiology and Economics, University of Groningen, Groningen, The Netherlands

${ }^{4}$ Helen \& Douglas House, Oxford, UK

${ }^{5}$ John Radcliffe Hospital, Oxford University Hospitals NHS Foundation Trust, Oxford, UK

${ }^{6}$ Department of Public Health and Primary Care, University of Cambridge, Cambridge, UK
}

${ }^{7}$ Great Ormond Street Hospital for Children NHS Foundation Trust, London, UK

${ }^{8}$ Surrey Clinical Trials Unit, University of Surrey, Surrey, UK

${ }^{9}$ Research Department of Pratice \& Policy, University College London School of Pharmacy, London, UK

${ }^{10} \mathrm{Centre}$ for Medicines Optimisation Research and Education, University College London School of Pharmacy and University College London Hospital, London, UK

${ }^{11}$ School of Psychology, University of Southampton, Southampton, UK

Corresponding author:

Christina Liossi, School of Psychology, University of Southampton, Highfield, Southampton SO17 1BJ, UK.

Email: cliossi@soton.ac.uk 


\section{What is already known about the topic?}

- Pain is a well-documented, highly prevalent symptom in infants, children and young people with life-limiting conditions.

- Assessing pain in infants, children and young people remains a challenge due to diverse patient conditions, types of pain and often a reduced ability or inability of patients to communicate verbally.

- No pain assessment tools are validated specifically for use within paediatric palliative care.

\section{What this paper adds?}

- This systematic review found no evidence of pain assessment tools that have been explicitly validated for the paediatric palliative care setting.

- The Faces Pain Scale-Revised (FPS-R) is recommended for self-assessment, and The Faces, Legs, Activity, Cry and Consolability scale (FLACC)/FLACC-Revised and Paediatric Pain Profile (PPP) are the recommended observational tools in their respective validated age groups.

\section{Implications for practice, theory or policy}

- A number of scales demonstrated high feasibility but were not recommended due to the lack of validation evidence in infants, children and young people with life-limiting conditions in paediatric palliative care settings.

- Validation data of pain assessment tools is a prerequisite to selecting an optimal tool to effectively assess pain in this population.

- Robust clinical validation of pain assessment tools in paediatric palliative care settings is urgently needed for the longterm improvement of pain management and quality of life in children at the end-of-life.

\section{Introduction}

Connor et al. ${ }^{1}$ estimated a global prevalence of 21 million children who required paediatric palliative care. In England alone, there were approximately 86,625 infants, children and young people living with life-limiting conditions in 2017/18., ${ }^{2,3}$ Compared to adults, 'infants, children and young people' experience a wider range of life-limiting conditions with significantly greater prognostic uncertainties, unpredictable symptoms and variable timescales of disease progression. Hain et al., ${ }^{4}$ using International Classification of Diseases-10 codes, compiled a directory of nearly 400 conditions that could limit the life of infants, children and young people according to the categorisation and definitions provided by the Association for Children's Palliative Care/Royal College of Paediatrics and Child Health. ${ }^{5}$ Partly due to recent health technological advances, infants, children and young people with lifelimiting conditions experience significantly longer disease trajectories and thus require paediatric palliative care over extended periods.

The ultimate goal of paediatric palliative care, unquestionably, is to enhance the quality of life for infants, children and young people and their families, with a cornerstone of holistic care being the prevention, early identification, comprehensive assessment and management of pain and other distressing symptoms. ${ }^{6}$ Pain is one of the most distressing and prevalent end-of-life symptoms experienced by patients. ${ }^{7}$ In paediatric palliative care settings of infants, children and young people with progressive malignant diseases, pain was experienced in over
$70 \%$ to over $90 \%$ in the different populations from United States, ${ }^{8}$ Japan, ${ }^{9}$ Sweden ${ }^{10}$ and the United Kingdom. ${ }^{11}$ However, paediatric pain is particularly prone to underdiagnosis, and under- or suboptimal treatment. ${ }^{12,13}$ In the aforementioned American study, ${ }^{8}$ only $27 \%$ of all children who reported pain actually experienced pain relief. Despite practitioners' experience and self-efficacy regarding pain assessment, barriers such as the fear of side effects, abuse and inappropriate use remain around effective analgesia management. $7,14,15$

Paediatric pain of any aetiology is a biopsychosocial phenomenon ${ }^{16}$ and the palliative pain experience in particular, or 'total pain', is a multidimensional experience that includes physical, psychological, social, spiritual and practical dimensions. ${ }^{17}$ Even though pain assessment is a critical first step for adequate pain management across treatment settings ${ }^{18}$ and is advocated by national ${ }^{19}$ and international guidelines, ${ }^{20}$ assessing pain in paediatric palliative care remains a challenge. This is due to diverse patient conditions, types of pain and often a reduced ability or inability of patients to communicate verbally. Furthermore, despite the vast amount of pain assessment tools available, there is an absence of tools explicitly for use in paediatric palliative care for infants, children and young people with life-limiting conditions. Therefore, a suitable tool for use with this group must either be developed specifically or adapted from polyvalent tools that have been validated in populations that include infants, children and young people with life-limiting conditions in paediatric palliative care. In addition, a range of validated tools needs to be available to meet the different 
Table 1. Eligibility criteria according to the COSMIN guidelines ${ }^{30}$ for the systematic review of pain assessment tools used in paediatric palliative care.

\begin{tabular}{lll}
\hline Criteria & Inclusion criteria & Exclusion criteria \\
\hline Construct & Pain & The tool does not assess pain \\
Population & Infants, children and young people & The study sample (or an arbitrary majority, e.g. $\geqslant 50 \%$ ) \\
& aged 0-18 with life-limiting & does not represent infants, children and young people \\
& conditions & aged 0-18 with life-limiting conditions \\
Instrument & Pain assessment tools & Not applicable (all assessment tools are considered) \\
Psychometric properties & COSMIN defined Validity, Reliability, & The study does not aim to evaluate one or more \\
& Responsiveness, Interpretability, & psychometric properties of a pain assessment tool, its \\
& Acceptability Measures & development or its interpretability
\end{tabular}

developmental and communication needs of the paediatric palliative care population.

Numerous reviews have been conducted to summarise the development and clinical validation of paediatric pain assessment tools in various contexts. ${ }^{21-24} \mathrm{~A}$ recent systematic review of reviews by Andersen et al. ${ }^{25}$ identified 65 observational paediatric pain assessment tools. Birnie et al. ${ }^{26}$ evaluated 8 out of the 60 self-report pain intensity assessment tools identified. However, neither study focussed on paediatric palliative care settings. Paediatric palliative care settings may include a variety of care settings, such as any tertiary care facilities, emergency rooms, community health centres, hospice facilities or even the children's home that offer support to 'the care of children and families facing chronic life limiting illnesses'. ${ }^{27}$ Another review conducted by Batalha et al. ${ }^{28}$ identified 17 pain assessment tools that assessed pain in children with cancer but the review did not produce any conclusive recommendations regarding the optimal pain assessment tools for use in this population. Furthermore, the study excluded paediatric patients with cognitive impairment, limiting the generalisability of the findings to a paediatric palliative care population.

The aims of this systematic review were to evaluate the psychometric properties of current age-specific pain assessment tools validated in various populations of children with life-limiting and life-threatening illnesses receiving palliative care and make recommendations for clinical practice. More specifically we addressed the following review questions:

- What pain assessment tools have been used to assess pain in populations of children with life-limiting and life-threatening illnesses receiving palliative care?

- What are the psychometric properties of these tools? This includes the validity, reliability and responsiveness of the pain assessment tools according to the COnsensus-based Standards for the selection of health Measurement Instruments (COSMIN).

- What are the most suitable pain assessment tools for use in paediatric palliative care?

\section{Methods}

We undertook a systematic review and narrative synthesis of peer-reviewed studies published in full and in English since the inception of the respective electronic databases, PsycINFO via ProQuest, Web of Science Core, Medline via Ovid, EMBASE, BIOSIS and CINAHL, up to April 2021. The reference lists of included journal articles, and existing reviews were hand searched. The review was reported in accordance with the Preferred Reporting Items for Systematic reviews and Meta-Analyses (PRISMA) and the COnsensus-based Standards for the selection of health Measurement INstruments (COSMIN) guidelines. ${ }^{29,30}$

\section{Search strategy}

The search strategy was developed based on suggestions from the COSMIN guidelines, incorporating construct, population and instrument search together with the COSMIN psychometric properties filter (see Supplemental File 1). ${ }^{30-32}$ Combinations of keywords, text words, Medical Subject Headings (MeSH) and other terms relevant to the four components were chosen for each database to optimise the sensitivity and specificity of the search. The thesaurus vocabulary of each database was used to adapt the search terms. The search terms were then combined with the COSMIN search filters (available on http://www.cosmin.nl/). ${ }^{32}$ Also incorporated in the search strategy, were search filters such as that developed by the National Institute for Health and Care Excellence (NICE), the Palliative Care Search Filter (PCSF) and relevant systematic reviews conducted by Beecham et al. (2016) and Anderson et al. (2017). ${ }^{25,33-35}$

\section{Inclusion/exclusion criteria}

Study eligibility criteria were developed according to the COSMIN guidelines ${ }^{30}$ (see Table 1). All published peerreviewed studies, regardless of study design, that reported the use of pain assessment tools in paediatric palliative settings completed by the parent or clinician of the infants, children and young people or the patient themselves were considered for inclusion in the review. Studies 
were excluded if they were not published in English, did not describe scientific research, were not peer-reviewed articles, or if they were not conducted in end-of-life care, palliative care or hospice care settings. We also excluded studies that used an assessment tool that could not be replicated due to the lack of clarity in methodology, version used or statistics.

\section{Extraction}

Two researchers ( $A C$ and $M G$ ) independently assessed study eligibility and undertook data extractions simultaneously. A standardised data extraction form modified from the COSMIN guidelines ${ }^{30}$ was used to record information on the context, population and outcomes and psychometric properties of each study. The COSMIN taxonomy, terminology and definitions of measurement properties for health-related patient-reported outcomes was used to appraise the psychometric properties of the instruments reviewed. ${ }^{36}$ COSMIN provides a consensus on terminology surrounding psychometric properties and a checklist for evaluating the methodological quality of studies reporting on validity, reliability and responsiveness. ${ }^{37}$ To synthesise the results from the included studies, the following data were extracted from each paper and organised into tables: authors, year and country of publication, journal, aim, sample size and characteristics, study setting, study design, measure(s), outcomes and psychometric properties.

\section{Risk of bias and quality of the results assessment of individual studies}

The methodological quality of each included study was assessed according to the COSMIN Risk of Bias Checklist. ${ }^{38}$ For all studies, each psychometric property was rated against the standards of quality listed in boxes 3-10 of the checklist to screen for methodological flaws that could lead to bias. Each question in the respective boxes was given the answer 'Very good', 'Adequate', 'Doubtful', 'Inadequate' or 'Not applicable', on the scoring form provided on the COSMIN website. ${ }^{32}$ Combining the individual answers on 98 items (5-18 items per psychometric property), each psychometric property per study was given a rating of 'Verygood', 'Adequate', 'Doubtful' or 'Inadequate'. As there is no consensus on the gold standard for pain assessment tools in paediatric palliative care settings currently, all self-report measures are considered the gold standard when assessing criterion validity, which assesses the degree to which the measures are an adequate reflection of a 'gold standard'.

Each psychometric property per study was rated against the latest criteria for good psychometric properties on the COSMIN guidelines (Table 2 ) $^{30}$ and was then given a rating of 'sufficient' $(+)$, 'insufficient' $(-)$ or 'indeterminate' (?).

\section{Data synthesis and risk of bias across studies}

Due to the heterogeneity of the studies, the results were qualitatively summarised together with considerations of the quantitative significance of each finding. Quantitative pooling of results was not conducted. The summarised evidence of psychometric properties per tool was rated against the same criteria for good psychometric properties with regards to the strength of evidence. The risks of bias across studies were graded using the Grades of Recommendation, Assessment, Development and Evaluation (GRADE) approach, where summarised evidence was downgraded based on five factors of insufficient quality of evidence. These were: risk of bias, inconsistency of results of studies, indirectness of populations validated compared to our population of interest and imprecision of results. ${ }^{38,39}$ Initial ratings from $A C$ and GG had fair interrater agreement (Kappa $=0.39$ for psychometric property results and 0.30 for GRADE ratings). Discrepancies in results were further discussed in a consensus meeting (between $A C$ and $G G$ ) where absolute agreement was reached.

The final recommendations for clinical practice and research were discussed and agreed by both clinicians and researchers, co-authors $A C, E J, C L, R H, E H, I W$ who are members of the DIPPER study. The DIPPER study is a fourphase feasibility study of a randomised clinical trial of transmucosal diamorphine versus oral morphine for breakthrough pain in children and young people with lifelimiting conditions.

\section{Results}

Figure 1 presents the flow of studies through the review. The initial search yielded 1157 articles with a further 11 articles from the manual search of reference lists of included studies. A total of 1168 articles were assessed for eligibility. After removing 199 duplicates, 969 titles and abstracts were screened against the eligibility criteria. Two hundred and one full-text articles were retrieved and reviewed independently by two researchers ( $A C$ and $G G$ ).

One hundred and sixty-seven articles were further excluded either because they did not describe a tool that measured pain $(n=25)$, the study sample did not represent a paediatric palliative care population $(n=73)$ or the study did not evaluate any psychometric properties $(n=69)$. A total of 34 articles were included in the review.

\section{Pain measurement tools and study populations}

A total of 22 pain assessment tools were evaluated in this review. Symptoms Screening in Paediatrics (SSPedi) 
Table 2. Psychometric properties recorded according to COSMIN guidelines.

\begin{tabular}{|c|c|c|c|}
\hline Domain & $\begin{array}{l}\text { Psychometric } \\
\text { property }\end{array}$ & $\begin{array}{l}\text { Aspect of a } \\
\text { psychometric property }\end{array}$ & Definition \\
\hline Reliability & & & $\begin{array}{l}\text { The degree to which the measurement is free from measurement } \\
\text { error }\end{array}$ \\
\hline \multirow[t]{4}{*}{$\begin{array}{l}\text { Reliability } \\
\text { (extended } \\
\text { definition) }\end{array}$} & & & $\begin{array}{l}\text { The extent to which scores for patients who have not changed are } \\
\text { the same for repeated measurement under several conditions: for } \\
\text { example using different sets of items from the same PROM (internal } \\
\text { consistency); over time (test-retest); by different persons on the } \\
\text { same occasion (inter-rater); or by the same persons (i.e. raters or } \\
\text { responders) on different occasions (intra-rater) }\end{array}$ \\
\hline & $\begin{array}{l}\text { Internal } \\
\text { consistency }\end{array}$ & & The degree of the interrelatedness among the items \\
\hline & Reliability & & $\begin{array}{l}\text { The proportion of the total variance in the measurements which is } \\
\text { due to 'true' differences between patients }\end{array}$ \\
\hline & $\begin{array}{l}\text { Measurement } \\
\text { error }\end{array}$ & & $\begin{array}{l}\text { The systematic and random error of a patient's score that is not } \\
\text { attributed to true changes in the construct to be measured }\end{array}$ \\
\hline \multirow[t]{8}{*}{ Validity } & & & $\begin{array}{l}\text { The degree to which a PROM measures the construct(s) it purports to } \\
\text { measure }\end{array}$ \\
\hline & Content validity & & $\begin{array}{l}\text { The degree to which the content of a PROM is an adequate reflection } \\
\text { of the construct to be measured }\end{array}$ \\
\hline & & Face validity & $\begin{array}{l}\text { The degree to which (the items of) a PROM indeed looks as though } \\
\text { they are an adequate reflection of the construct to be measured }\end{array}$ \\
\hline & $\begin{array}{l}\text { Construct } \\
\text { validity }\end{array}$ & & $\begin{array}{l}\text { The degree to which the scores of a PROM are consistent with } \\
\text { hypotheses (for instance with regard to internal relationships, } \\
\text { relationships to scores of other instruments or differences between } \\
\text { relevant groups) based on the assumption that the PROM validly } \\
\text { measures the construct to be measured }\end{array}$ \\
\hline & & Structural validity & $\begin{array}{l}\text { The degree to which the scores of a PROM are an adequate reflection } \\
\text { of the dimensionality of the construct to be measured }\end{array}$ \\
\hline & & Hypotheses testing & Item construct validity \\
\hline & & Cross-cultural validity & $\begin{array}{l}\text { The degree to which the performance of the items on a translated } \\
\text { or culturally adapted PROM are an adequate reflection of the } \\
\text { performance of the items of the original version of the PROM }\end{array}$ \\
\hline & $\begin{array}{l}\text { Criterion } \\
\text { validity }\end{array}$ & & $\begin{array}{l}\text { The degree to which the scores of a PROM are an adequate reflection } \\
\text { of a 'gold standard' }\end{array}$ \\
\hline Responsiveness & & & $\begin{array}{l}\text { The ability of a PROM to detect change over time in the construct to } \\
\text { be measured }\end{array}$ \\
\hline & Responsiveness & & Item responsiveness \\
\hline Interpretability* & & & $\begin{array}{l}\text { Interpretability is the degree to which one can assign qualitative } \\
\text { meaning - that is, clinical or commonly understood connotations - to } \\
\text { a PROM's quantitative scores or change in scores. }\end{array}$ \\
\hline
\end{tabular}

*Although an important property of an instrument, interpretability is not a psychometric property.

had both self-report and proxy report versions. Characteristics of all included measures and populations are listed in Table 3 for observational and selfreport tools. Apart from the Oral Mucositis Daily Questionnaire (OMDQ), 40 none of the measures included were disease-specific. Mirroring the distribution of life-limiting conditions in paediatric palliative care, the majority of the tools were developed, or validated, in children with cancer. Other populations included children with haematological, neurological and surgical conditions, and those requiring intensive care.

\section{Feasibility of use}

Feasibility of use of tools was assessed by evaluating their respective types and ease of administration, completion time, training time, recall time, length of the instrument, ease of scoring, calculation and standardisation. Given the diversity and needs in patients with life-limiting conditions, there is no single feasible tool.

Completion time was reported in all of the self-report tools apart from the paediatric pain profile (PPP), and ranged from 1 to $10 \mathrm{~min}$. Completion time was not reported for most observational tools. Training time and 


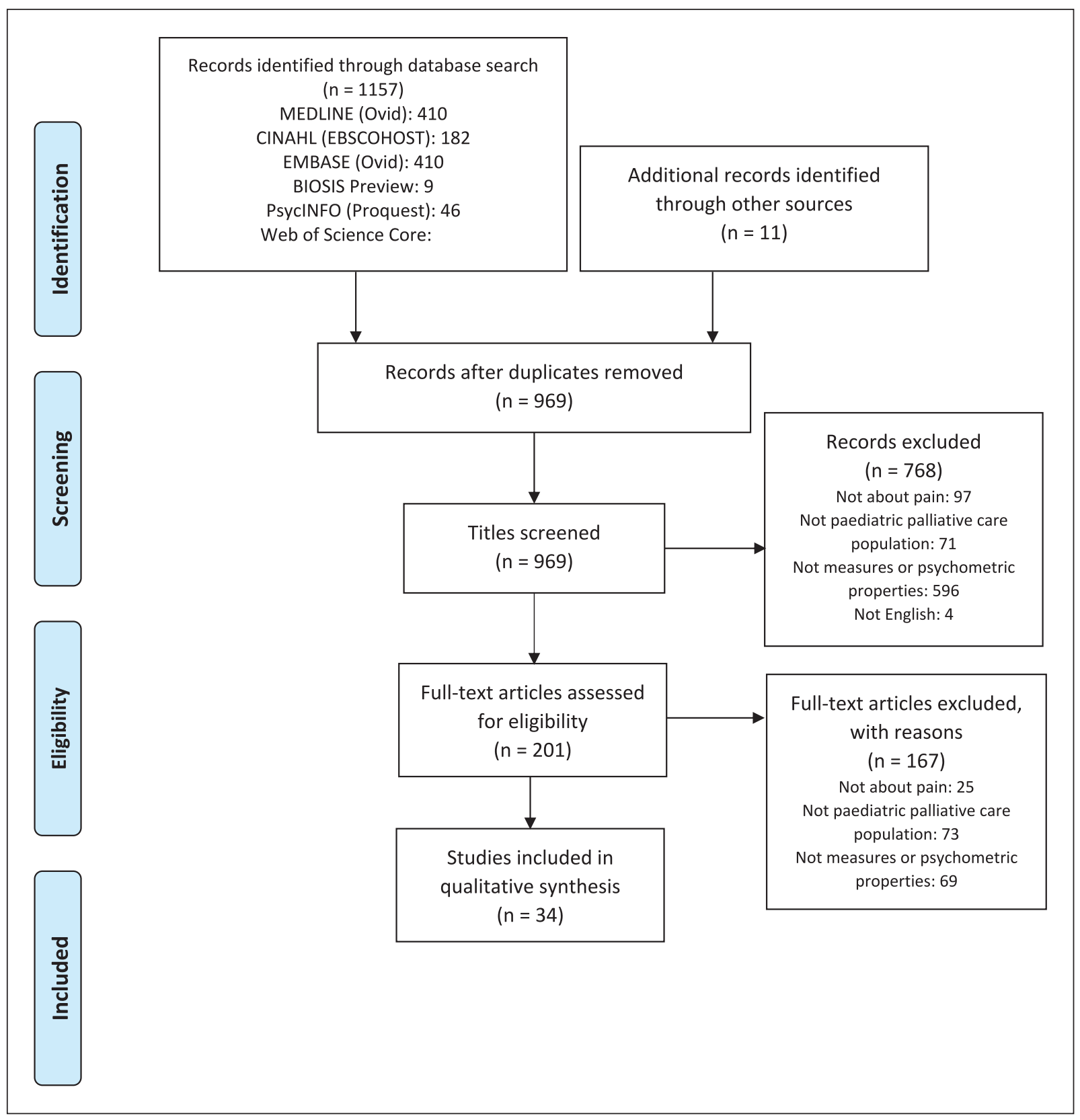

Figure 1. PRISMA flow diagram of records identified in the systematic review of pain assessment tools used in paediatric palliative care.

recall period were generally not very well reported. Most tools adopt point scales or other forms of ordinal responses, with the adolescent paediatric pain tool (APPT), children's procedural interview (CPI) and Pain Squad incorporating semi- structured or open answers in the response options. All tools that used an ordinal response system were relatively easier to standardise, calculate and administer. Of all reported tools, the Pain Squad and the Symptoms Screening in Paediatrics (SSPedi) were developed as electronic versions to be administered using mobile devices.

\section{Methodological quality}

Ratings for methodological quality of psychometric properties per study are illustrated in Supplemental File 2. Internal structure was assessed by the reporting of content validity, structural validity, cross-cultural validity and internal consistency. Internal consistency was the property most frequently reported to demonstrate internal structure of each tool. Most studies also described hypothesis testing properties, convergent and divergent validity. Although reliability measures were often reported, when referencing dichotomous, nominal or ordinal scores, a 


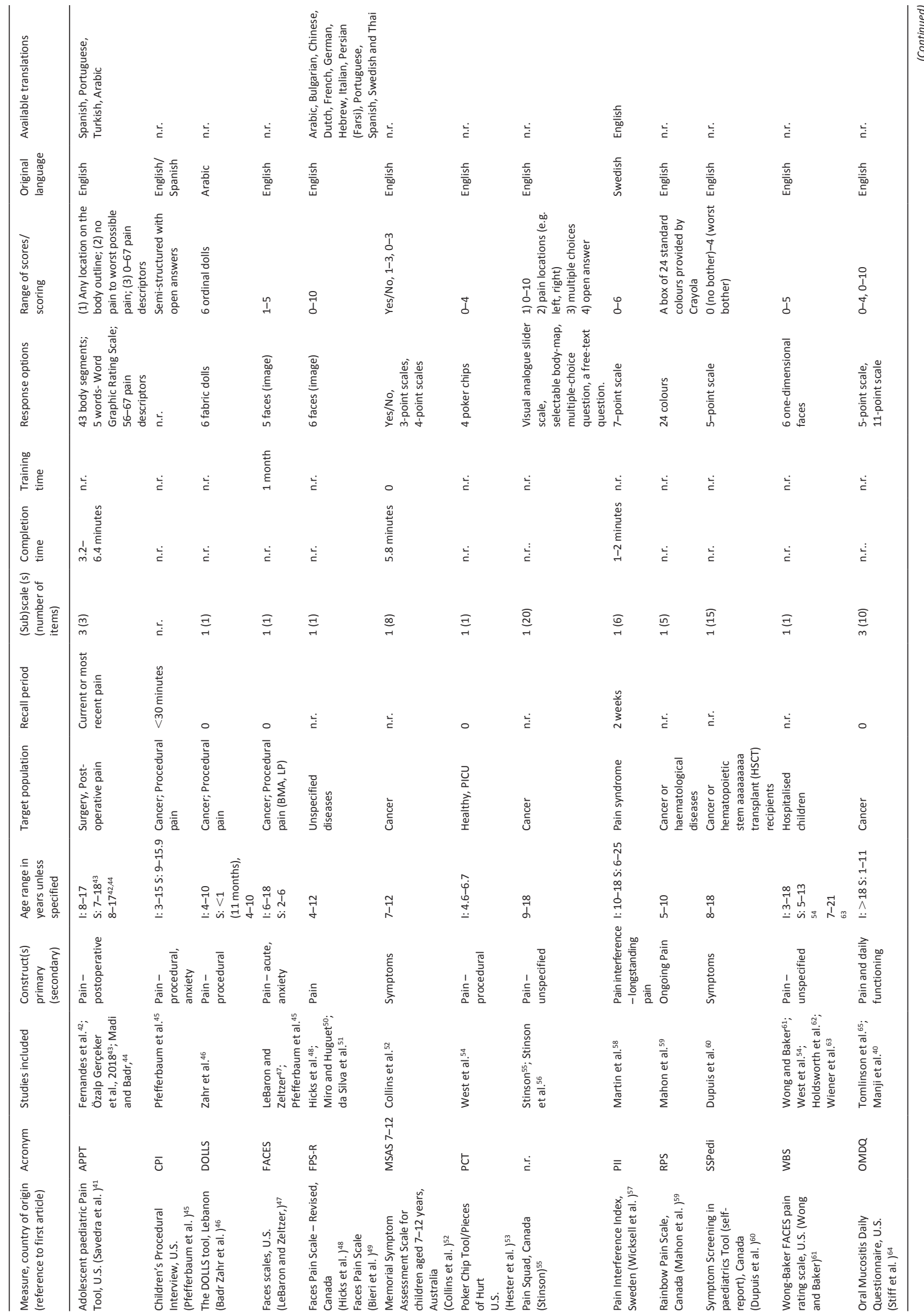




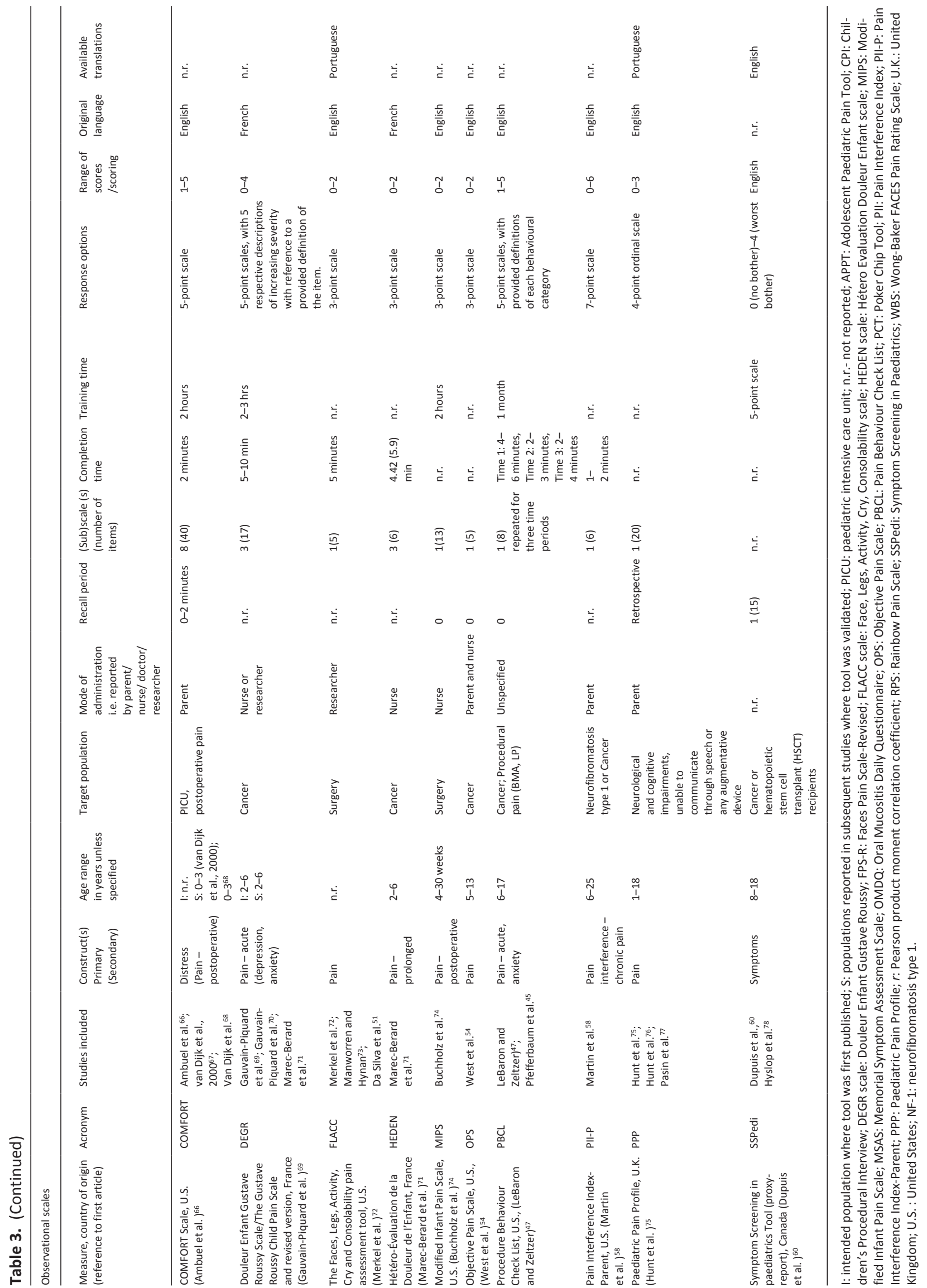


number of studies failed to demonstrate evidence of kappa calculations and weighting schemes, ${ }^{43}$ while some only reported the Pearson or Spearman correlation coefficient without any kappa calculation. ${ }^{40,65,71}$ This significantly discounted the strength of reported evidence. Only one study referred to intrarater agreement. ${ }^{76}$

Most of the included studies provided limited evidence of psychometric properties in their respective populations, none reported information on all four domains or more than half of the psychometric properties of interest. Psychometric property results and respective methodological quality ratings of individual studies are summarised in Supplemental File 2. A summary of the qualitatively pooled findings can be found in Table 4.

Content validity was assessed in five of the studies for four of the tools and generally there was appropriate evaluation on relevance and comprehensibility in patients and experts. Structural validity was assessed in four of the studies for two of the tools and generally there was appropriate use of confirmatory or exploratory factor analysis except for one study which used Principal Component Analysis. ${ }^{66}$ Cross cultural validity was assessed in four of the studies for three of the tools utilising either independent translation, back-translation or expert committee. Internal consistency was assessed in 14 of the studies for 16 of the tools and generally there was appropriate use of Cronbach's alpha. Hypothesis testing was assessed in all of the tools except $\mathrm{RPS}^{59}$ and generally there was appropriate use of convergent and divergent validity. Criterion validity was assessed in 14 of the studies for 14 of the tools and generally there were assessments on either concurrent or predictive validity. Reliability was assessed in 19 of the studies for 14 of the tools and in general there was appropriate use of test-retest reliability, interrater agreement and intrarater agreement.

\section{Pain assessment tools recommended for clinical practice}

\section{Self-report}

The faces pain scale-revised. The Faces Pain ScaleRevised (FPS-R) consists of six drawings of faces, arranged in a horizontal row, with a neutral face at the left (score of 0 ) and the maximum pain face at the right (score of 10). The tool was validated in a clinical sample of 5- to 17-yearolds who were admitted to the hospital with cancer. Brazilian and Catalan versions of the tool were examined. The tool demonstrated strong evidence of criterion and construct validity. The internal structure of the tool was not well examined. The FPS-R cross-cultural validity was demonstrated with the development process of the Catalan version. However, the tool was not validated culturally after the translation process.

\section{Observational tools}

The faces, legs, activity, cry and consolability scale (FLACC). The FLACC scale measures pain intensity by rating five behaviours (face, legs, activity, consolability and cry) each scored from 0 to 2 to derive a total score from 0 to 10 . The descriptors for each item are considered indicative of behaviours exhibited by children in pain and the descriptors associated with each score level $(0,1$ or 2$)$ seen to represent an escalation consistent with increasing pain intensity. The tool was initially developed to measure distress and widely adopted for measuring post-operative pain. It was validated in 4- to 17-year-olds. English, Arabic and Brazilian Portuguese versions are examined in this review. The tool has good internal consistency and reliability. The Brazilian Portuguese version of the FLACC demonstrated sufficient internal consistency in 7- to 17-year-olds; other measures of internal structure were not reported. The original English version possesses high interrater agreement.

Paediatric pain profile. The Paediatric Pain Profile (PPP) is a 20-item rating scale. Each item is rated on a fourpoint scale as occurring 'not at all' to 'a great deal' in any given time period. The total score ranges from 0 to 60 . The tool is validated in 1- to 18-year-olds with neurological and cognitive impairment. In the Brazilian version of the PPP, content validation was conducted with health professionals and primary caregivers. The consensus yielded a clarity rating between clear and very clear. The tool has very good criterion validity when using the numerical rating scale as a gold standard. Reliability was assessed in terms of interrater agreement, interrater agreement and test-retest reliability in various studies. Overall, the tool has good reliability, good internal consistency and insufficient convergent validity with physiological measures.

\section{Discussion}

\section{Main findings}

This is the first systematic review of pain assessment tools currently being used in paediatric palliative care, together with an examination of their psychometric properties and feasibility allowing us to make recommendations for clinical practice. Balancing aspects of feasibility and psychometric properties, the FPS- $R$ is the recommended self-report measure while PPP and FLACC are the recommended observational tools. The revised FLACC $\mathrm{R}^{79}$ scale is an observational pain tool based on the FLACC scale modified to include additional pain behaviours often found in children who are non-verbal or with cognitive impairment. It includes additional items reported by parents related to their child's individualised behaviours within each category. Although no studies in this review 


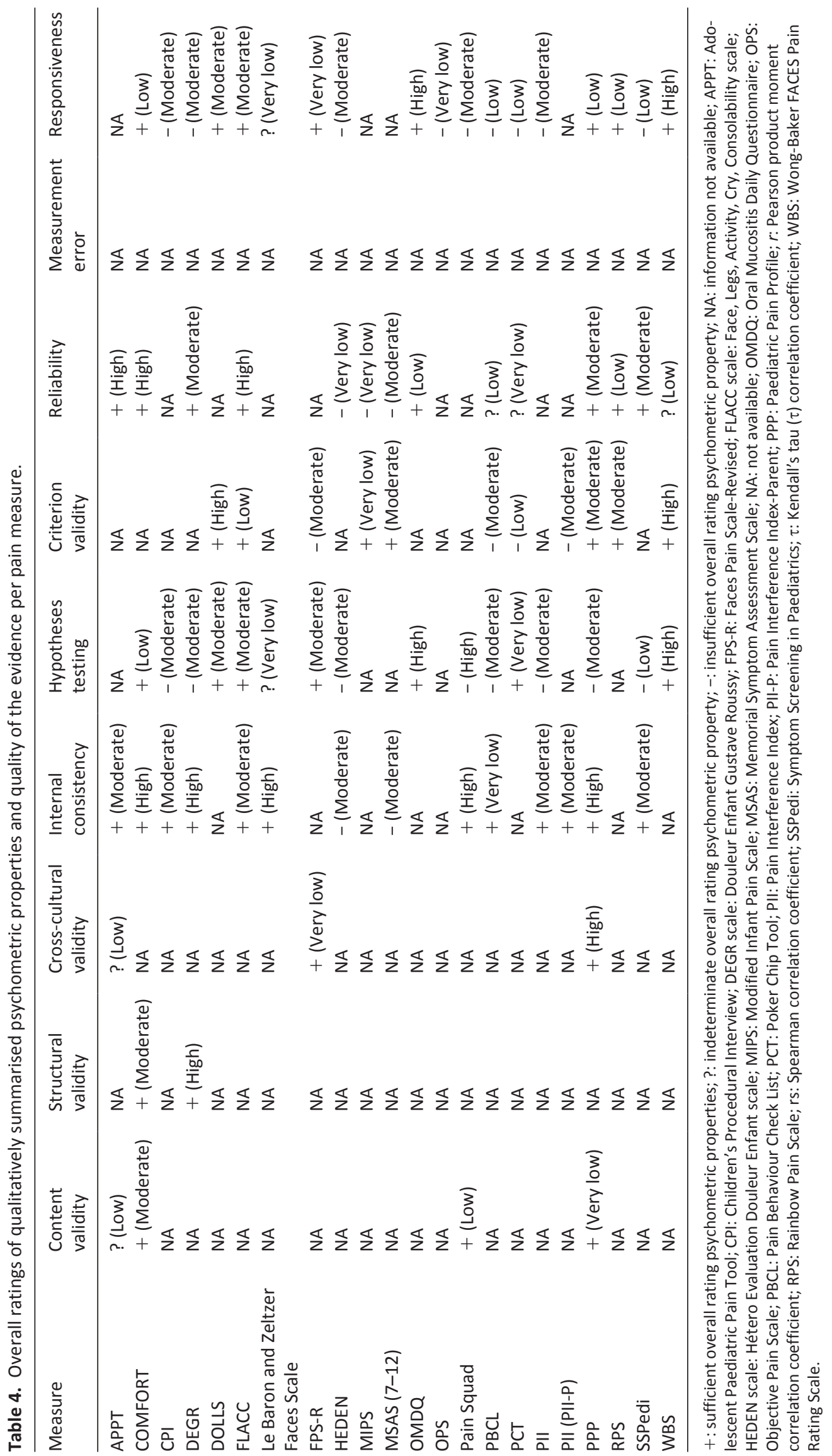


have included FLACC $R$, we recommend it for children who are non-verbal or with cognitive impairment.

As pain is a subjective experience, whenever possible, self-report from infants, children and young people should be the first-choice method of pain assessment. ${ }^{18}$ Infants, children and young people with life-limiting conditions are often cared for in non-clinical settings, therefore parent or carer reports should be regarded as the next choice of pain assessment. Our recommendations prioritise tools that have been validated with a high correlation between parent and self-report. We recommend all pain assessment tools that demonstrated sufficient content validity and internal consistency in infants, children and young people with life-limiting conditions population where results truly reflect patients' pain intensity. As indicated by the COSMIN guidelines, measures with high quality evidence for insufficient psychometric properties are not recommended for use in the defined target population, infants, children and young people with lifelimiting conditions, until a modified version of the tool is proven valid and reliable in that population.

\section{Strengths and limitations}

This is the first systematic review that has followed an established methodology to summarise and critically appraise the extant literature pertaining to the reliability, validity, responsiveness and feasibility of pain assessment tools in paediatric palliative care. Clinicians routinely need to make complex decisions and identify the appropriate pain measurement tool for different patient populations. Systematic reviews of psychometric properties provide the necessary evidence base to underpin these decisions.

\section{What this study adds}

In their review, Coombes et al., ${ }^{80}$ reported a range of challenges associated with the inherent limitations of the COSMIN checklist, including but not limited to, the content validation process of the checklist, its interrater reliability and the ambiguity of inadequate reporting and inadequate quality of each psychometric property.

This review examines the feasibility of pain measures by looking at each tool's intrinsic characteristics (Table 3) such as recall period, completion time, number of items, mode of administration, settings in which the tool has been validated and other factors. Due to insufficient information available from the existing literature, other extrinsic relevant aspects were not thoroughly examined. These include: patient's and clinicians' comprehensibility, copyright and regulatory agency's requirement for approval. Interpretability was not examined in pain tools due to the fact that most tools aimed to measure one dimension of pain that is, pain intensity.

By default, pain measurement tools are developed to help standardise reporting and inform clinicians and carers of pain management decisions in a way that is generalisable and comprehensible to a third party who is not experiencing the pain. The pain tools recommended in this review were chosen as a result of weighing up the feasibility and psychometric properties of the tools. As such, the tools may not be optimal in the measurement of all types of pain experienced by infants, children and young people in palliative care such as breakthrough pain ${ }^{81}$ for example.

Clinical implications and future directions Despite the vast amount of pain assessment tools developed and the numerous studies on the utilisation of these tools, our search found few that focussed on the validation of these tools in infants, children and young people with life-limiting conditions in paediatric palliative care settings. A number of scales demonstrated high levels of feasibility but were not recommended due to the lack of validation evidence in infants, children and young people with life-limiting conditions or paediatric palliative care settings. Validation data of pain assessment tools is a prerequisite to the selection of an optimal tool to effectively assess pain in this population. Given the significant distress caused by pain, and its effect on the quality of life of children particularly at the end-of-life, implementation and standardisation of pain assessment is urgently needed. This calls for robust clinical validation efforts in paediatric palliative care settings for the long-term improvement of pain management.

\section{Author's note}

Kate Oulton is a National Institute for Health Research (NIHR) Senior Nurse and Midwife Research Leader.

\section{Author contributions}

IW is the Chief Investigator of the DIPPER study and conceived the project and takes overall responsibility for the conduct of the study. AYLC was involved in the study design, acquisition, data screening, data extraction, quality assessment, qualitative synthesis or interpretation of data and drafted the manuscript with input from all authors. GMG was involved in the cross checking of data screening, data extraction, quality assessment and qualitative synthesis. LJ was involved in planning and study design, contributed to the search strategy and commented on various drafts of the manuscript. All authors approved the final manuscript as submitted and agree to be accountable for all aspects of the work.

\section{Declaration of conflicting interests}

The author(s) declared no potential conflicts of interest with respect to the research, authorship, and/or publication of this article.

\section{Funding}

The author(s) disclosed receipt of the following financial support for the research, authorship, and/or publication of this article: This project is funded by the National Institute for Health Research (NIHR) under its Research for Patient Benefit (RfPB) Programme (Grant Reference Number PB-PG-0317-20036). 
The views expressed are those of the author(s) and not necessarily those of the NIHR or the Department of Health and Social Care.

\section{ORCID iDs}

Simon S Skene (iD https://orcid.org/0000-0002-7828-3122

Liz Jamieson (iD https://orcid.org/0000-0002-3667-0423

Richard F Howard (iD https://orcid.org/0000-0001-9271-0074

Christina Liossi iD https://orcid.org/0000-0003-0627-6377

\section{Supplemental material}

Supplemental material for this article is available online.

\section{References}

1. Connor SR, Downing J and Marston J. Estimating the global need for palliative care for children: a cross-sectional analysis. J Pain Symptom Manag 2017; 53: 171-177.

2. Fraser LK, Gibson-Smith D, Jarvis S, et al. 'Make Every Child Count' estimating current and future prevalence of children and young people with life-limiting conditions in the United Kingdom. https://www.togetherforshortlives. org.uk/wp-content/uploads/2020/04/PrevalencereportFinal_28_04_2020.pdf (2020, accessed December 19 2020).

3. National Guideline Alliance (UK). End of life care for infants, children and young people with life-limiting conditions: planning and management. London: National Institute for Health and Care Excellence (UK, 2016.

4. Hain R, Devins $M$, Hastings $R$, et al. Paediatric palliative care: development and pilot study of a 'Directory' of lifelimiting conditions. BMC Palliat Care 2013; 12: 43.

5. ACT/RCPCH. A guide to the development of children's palliative care services. Bristol and London, 1997. ACT/RCPCH.

6. Radbruch L, De Lima L, Knaul F, et al. Redefining palliative care-A new consensus-based definition. J Pain Symptom Manag 2020; 60: 754-764.

7. Grégoire $\mathrm{MC}$ and Frager $\mathrm{G}$. Ensuring pain relief for children at the end of life. Pain Res Manag 2006; 11: 163-171.

8. Wolfe J, Grier HE, Klar N, et al. Symptoms and suffering at the end of life in children with cancer. New Engl J Med 2000; 342: 326-333.

9. Hongo $\mathrm{T}$, Watanabe $\mathrm{C}, \mathrm{Okada} \mathrm{S}$, et al. Analysis of the circumstances at the end of life in children with cancer: symptoms, suffering and acceptance. Pediatr Int 2003; 45: 60-64.

10. Jalmsell L. Symptoms affecting children with malignancies during the last month of life: a nationwide follow-up. Pediatrics 2006; 117: 1314-1320.

11. Goldman A, Hewitt M, Collins GS, et al. Symptoms in children/young people with progressive malignant disease: United Kingdom children's Cancer Study Group/Paediatric Oncology nurses forum survey. Pediatrics 2006; 117: e1179-e1186.

12. Oostendorp LJ, Rajapakse D, Kelly P, et al. Documentation of breakthrough pain in narrative clinical records of children with life-limiting conditions: feasibility of a retrospective review. J Child Health Care 2019; 23: 564-578.
13. Mathews L. Pain in children: neglected, unaddressed and mismanaged. Indian J Palliat Care 2011; 17: 70-73.

14. Galloway KS and Yaster M. Pain and symptom control in terminally ill children. Pediatr Clin North Am 2000; 47: 711746.

15. Liben S. Pediatric palliative medicine: obstacles to overcome. J Palliat Care 1996; 12: 24-28.

16. Liossi $\mathrm{C}$ and Howard RF. Pediatric chronic pain: biopsychosocial assessment and formulation. Pediatrics 2016; 138: e20160331.

17. Ong CK and Forbes D. Embracing Cicely Saunders's concept of total pain. BMJ 2005; 331: 576.5-57577.

18. Howard RF and Liossi C. Pain assessment in children. Arch Dis Child 2014; 99: 1123-1124.

19. National Institute for Health and Care Excellence. End of life care for infants, children and young people with life-limiting conditions: planning and management. London, 2016. National Guideline Alliance (UK).

20. McGrath PJ, Walco GA, Turk DC, et al. Core outcome domains and measures for pediatric acute and chronic/ recurrent pain clinical trials: PedIMMPACT recommendations. J Pain 2008; 9: 771-783.

21. Quinn BL, Seibold E and Hayman L. Pain assessment in children with special needs: a review of the literature. Except Child 2015; 82: 44-57.

22. Lee RR, Rashid A, Ghio D, et al. Chronic pain assessments in children and adolescents: a systematic literature review of the selection, administration, interpretation, and reporting of unidimensional pain intensity scales. Pain Res Manag 2017; 2017: 1-17.

23. Stinson JN, Kavanagh T, Yamada J, et al. Systematic review of the psychometric properties, interpretability and feasibility of self-report pain intensity measures for use in clinical trials in children and adolescents. Pain 2006; 125: 143-157.

24. von Baeyer CL and Spagrud LJ. Systematic review of observational (behavioral) measures of pain for children and adolescents aged 3 to 18 years. Pain 2007; 127: 140-150.

25. Andersen RD, Langius-Eklöf A, Nakstad B, et al. The measurement properties of pediatric observational pain scales: a systematic review of reviews. Int J Nurs Stud 2017; 73: 93-101.

26. Birnie KA, Hundert AS, Lalloo C, et al. Recommendations for selection of self-report pain intensity measures in children and adolescents: a systematic review and quality assessment of measurement properties. Pain 2019; 160: 5-18.

27. Muckaden $M$, Balaji $P$, Tilve $P$, et al. Paediatric palliative care: theory to practice. Indian J Palliat Care 2011; 17: 52-60.

28. Batalha L, Fernandes A, Campos C, et al. Pain assessment in children with cancer: a systematic review. Revista de Enfermagem Referência 2015; №5: 119-127.

29. Moher D, Liberati A, Tetzlaff J, et al. Preferred reporting items for systematic reviews and meta-analyses: the PRISMA statement. BMJ 2009; 339: b2535-b2535.

30. Prinsen CAC, Mokkink LB, Bouter LM, et al. COSMIN guideline for systematic reviews of patient-reported outcome measures. Qual Life Res 2018; 27: 1147-1157.

31. Terwee CB, Jansma EP, Riphagen II, et al. Development of a methodological PubMed search filter for finding studies 
on measurement properties of measurement instruments. Qual Life Res 2009; 18: 1115-1123.

32. COnsensus-based standards for the selection of health measurement INstruments (COSMIN) website.

33. Aidoo $E$ and Rajapakse D. End of life care for infants, children and young people with life-limiting conditions: planning and management: the NICE guideline 2016. Arch Dis Child 2018; 103: 296-299.

34. Sladek R, Tieman J, Fazekas BS, et al. Development of a subject search filter to find information relevant to palliative care in the general medical literature. J Med Libr Assoc 2006; 94: 394-401.

35. Beecham E, Candy B, Howard R, et al. Pharmacological interventions for pain in children and adolescents with life-limiting conditions. Cochrane Database Syst Rev 2015. Issue 3. Art. No.: CD010750. DOI: 10.1002/14651858.CD010750.pub2

36. Mokkink LB, Terwee CB, Patrick DL, et al. The COSMIN study reached international consensus on taxonomy, terminology, and definitions of measurement properties for health-related patient-reported outcomes. J Clin Epidemiol 2010; 63: 737-745.

37. Mokkink LB, Terwee CB, Patrick DL, et al. The COSMIN checklist for assessing the methodological quality of studies on measurement properties of health status measurement instruments: an international Delphi study. Qual Life Res 2010; 19: 539-549.

38. Mokkink LB, de Vet HCW, Prinsen CAC, et al. COSMIN risk of bias checklist for systematic reviews of patient-reported outcome measures. Qual Life Res 2018; 27: 1171-1179.

39. Schünemann HJ, Mustafa R, Brozek J, et al. GRADE guidelines: 16. GRADE evidence to decision frameworks for tests in clinical practice and public health. J Clin Epidemiol 2016; 76: 89-98.

40. Manji A, Tomlinson D, Ethier MC, et al. Psychometric properties of the oral mucositis daily questionnaire for child self-report and importance of mucositis in children treated with chemotherapy. Support Care Cancer 2012; 20: 12511258.

41. Savedra MC, Holzemer WL, Tesler MD, et al. Assessment of postoperation pain in children and adolescents using the adolescent pediatric pain tool. Nurs Res 1993; 42: 5???9-9.

42. Fernandes $A$, Batalha $L$, Perdigão $A$, et al. Cultural validation of the adolescent pediatric pain tool (APPT) in portuguese children with cancer. Revista de Enfermagem Referência 2015; i série: 99-105.

43. Özalp Gerçeker G, Bilsin E, Binay Ş, et al. Cultural adaptation of the adolescent pediatric pain tool in Turkish children with cancer. Eur J Oncol Nurs 2018; 34: 28-34.

44. Madi D and Badr LK. Translation, cross-cultural adaptation, and validation of the adolescent pediatric pain tool (APPT) for multidimensional measurement of pain in children and adolescents. Pain Manag Nurs 2019; 20: 549-555.

45. Pfefferbaum B, Adams J and Aceves J. The influence of culture on pain in Anglo and Hispanic children with cancer. $J$ Am Acad Child Adolesc Psychiatry 1990; 29: 642-647.

46. Zahr LKB, Puzantian $\mathrm{H}$, Abboud M, et al. Assessing procedural pain in children with cancer in Beirut, Lebanon. $J$ Pediatr Oncol Nurs 2006; 23: 311-320.

47. LeBaron $S$ and Zeltzer L. Assessment of acute pain and anxiety in children and adolescents by self-reports, observer reports, and a behavior checklist. J Consult Clin Psychol 1984; 52: 729-738.

48. Hicks $\mathrm{CL}$, von Baeyer $\mathrm{CL}$, Spafford $\mathrm{PA}$, et al. The faces pain scale - revised: toward a common metric in pediatric pain measurement. Pain 2001; 93: 173-183.

49. Bieri D, Reeve RA, Champion DG, et al. The faces pain scale for the self-assessment of the severity of pain experienced by children: development, initial validation, and preliminary investigation for ratio scale properties. Pain 1990; 41: 139150.

50. Miró J and Huguet A. Evaluation of reliability, validity, and preference for a pediatric pain intensity scale: the Catalan version of the faces pain scale - revised. Pain 2004; 111: 59-64.

51. da Silva FC, Santos Thuler LC and de Leon-Casasola OA. Validity and reliability of two pain assessment tools in Brazilian children and adolescents. J Clin Nurs 2011; 20: 1842-1848.

52. Collins JJ, Devine TD, Dick GS, et al. The measurement of symptoms in young children with cancer: the validation of the memorial symptom assessment scale in children aged 7-12. J Pain Symptom Manag 2002; 23: 10-16.

53. Hester NO, Foster R and Kristensen K. Measurement of pain in children - generalizability and validity of the pain ladder and the poker chip tool. Adv Pain Res Ther 1990; 15: 79-84.

54. West N, Oakes L, Hinds PS, et al. Measuring pain in pediatric oncology ICU patients. . . Including commentary by Wong DL. J Pediatr Oncol Nurs 1994; 11: 64-68.

55. Stinson J. Pain assessment in children and youth with cancer: challenges and novel approaches. Pain Res Manag 2013; 18(2): e15.

56. Stinson J, Jibb LA, Nathan PC, et al. Construct validity and reliability of a multidimensional electronic pain diary for school-aged children and adolescents with cancer. Pediatr Blood Cancer 2015; 62(Supplement 4): S194.

57. Wicksell RK, Melin L, Lekander M, et al. Evaluating the effectiveness of exposure and acceptance strategies to improve functioning and quality of life in longstanding pediatric pain - aa randomized controled trial. Pain 2009; 141: 248-257.

58. Martin S, Nelson Schmitt S, Wolters PL, et al. Development and validation of the English pain interference index and pain interference index-parent report. Pain Med 2015; 16: 367-373.

59. Mahon P, Holsti L, Siden $\mathrm{H}$, et al. Using colors to assess pain in toddlers: validation of "The Rainbow Pain scale" -A proofof-principle study. J Pediatr Oncol Nurs 2015; 32: 40-46.

60. Dupuis LL, Johnston DL, Baggott C, et al. Validation of the symptom screening in pediatrics tool in children receiving cancer treatments. J Natl Cancer Inst 2018; 110: 661-668.

61. Wong $\mathrm{DL}$ and Baker $\mathrm{CM}$. Pain in children: comparison of assessment scales. Okla Nurse 1988; 33: 8.

62. Holdsworth MT, Raisch DW, Winter SS, et al. Differences among raters evaluating the success of EMLA cream in alleviating procedure-related pain in children with cancer. Pharmacotherapy 1997; 17: 1017-1022.

63. Wiener L, Battles H, Zadeh S, et al. Validity, specificity, feasibility and acceptability of a brief pediatric distress thermometer in outpatient clinics. Psychooncology 2017; 26(4): 461-468. 
64. Stiff PJ, Erder H, Bensinger WI, et al. Reliability and validity of a patient self-administered daily questionnaire to assess impact of oral mucositis (OM) on pain and daily functioning in patients undergoing autologous hematopoietic stem cell transplantation (HSCT). Bone Marrow Transplant 2006; 37: 393-401.

65. Tomlinson D, Ethier MC, Judd P, et al. Reliability and construct validity of the oral mucositis daily questionnaire in children with cancer. Eur J Cancer 2011; 47(3): 383-388.

66. Ambuel B, Hamlett KW, Marx CM, et al. Assessing distress in pediatric intensive care environments: the COMFORT scale. J Pediatr Psychol 1992; 17: 95-109.

67. van Dijk M, de Boer JB, Koot HM, et al. The reliability and validity of the COMFORT scale as a postoperative pain instrument in 0 to 3-year-old infants. Pain 2000; 84: 367377.

68. van Dijk M, de Boer JB, Koot HM, et al. The association between physiological and behavioral pain measures in 0to 3-year-old infants after major surgery. J Pain Symptom Manag 2001; 22: 600-609.

69. Gauvain-Piquard A, Rodary C, Rezvani A, et al. Pain in children aged 2-6 years: a new observational rating scale elaborated in a pediatric oncology unit - preliminary report. Pain 1987; 31: 177-188.

70. Gauvain-Piquard A, Rodary C, Rezvani A, et al. The development of the $D E G R(R)$ : a scale to assess pain in young children with cancer. Eur J Pain 1999; 3: 165-176.

71. Marec-Berard P, Gomez F, Combet S, et al. HEDEN pain scale: a shortened behavioral scale for assessment of prolonged cancer or postsurgical pain in children aged 2 to 6 Years. Pediatr Hematol Oncol 2015; 32: 291-303.

72. Merkel SI, Voepel-Lewis T, Shayevitz JR, et al. The FLACC: a behavioral scale for scoring postoperative pain in young children. Pediatr Nurs 1997; 23: 293-297.
73. Manworren RCB and Hynan LS. Practice applications of research. Clinical validation of FLACC: preverbal patient pain scale. Pediatr Nurs 2003; 29: 140-146.

74. Buchholz $M$, Karl HW, Pomietto $M$, et al. Pain scores in infants: a modified infant pain scale versus visual analogue. J Pain Symptom Manag 1998; 15: 117-124.

75. Hunt A, Goldman A, Seers K, et al. Clinical validation of the paediatric pain profile. Dev Med Child Neurol 2004; 46: 9-18.

76. Hunt A, Wisbeach A, Seers K, et al. Development of the paediatric pain profile: role of video analysis and saliva cortisol in validating a tool to assess pain in children with severe neurological disability. J Pain Symptom Manag 2007; 33: 276-289.

77. Pasin S, Avila F, de Cavatá T, et al. Cross-cultural translation and adaptation to Brazilian Portuguese of the paediatric pain profile in children with severe cerebral palsy. J Pain Symptom Manag 2013; 45: 120-128.

78. Hyslop S, Dupuis LL, Baggott C, et al. Validation of the proxy version of symptom screening in pediatrics tool in children receiving cancer treatments. J Pain Symptom Manag 2018; 56: 107-112.

79. Malviya S, Voepel-Lewis T, Burke C, et al. The revised FLACC observational pain tool: improved reliability and validity for pain assessment in children with cognitive impairment. Paediatr Anaesth 2006; 16: 258-265.

80. Coombes LH, Wiseman T, Lucas G, et al. Health-related quality-of-life outcome measures in paediatric palliative care: a systematic review of psychometric properties and feasibility of use. Palliat Med 2016; 30: 935-949.

81. Greenfield K, Holley S, Schoth DE, et al. A protocol for a systematic review and meta-analysis to identify measures of breakthrough pain and evaluate their psychometric properties. BMJ Open 2020; 10: e035541. 